Universidad de Lima

Facultad de Psicología

Carrera de Psicología

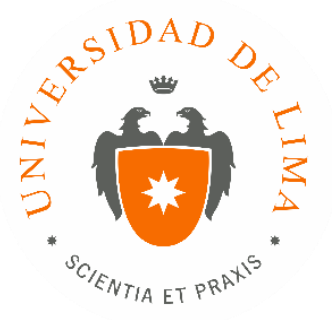

\title{
EXPERIENCIA EN INVESTIGACIÓN CUALITATIVA EN INSTITUTO DE INVESTIGACIÓN
}

Trabajo de suficiencia profesional para optar el título profesional de Licenciado en Psicología

Solana Macarena Scarafia

Código 20122217

Lima - Perú 
Febrero de 2019 


\section{EXPERIENCIA EN INVESTIGACIÓN CUALITATIVA EN INSTITUTO DE INVESTIGACIÓN}




\section{TABLA DE CONTENIDO}

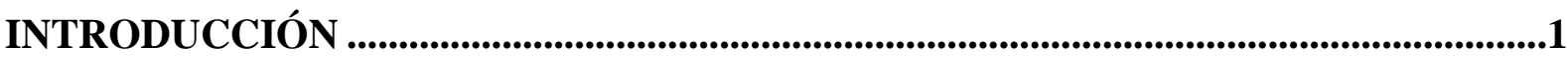

\section{CAPÍTULO I: IDENTIFICACIÓN DEL PROBLEMA ....................................................................2}

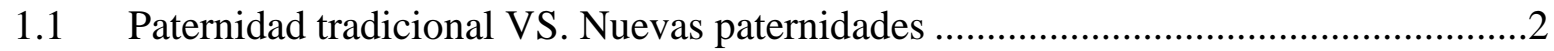

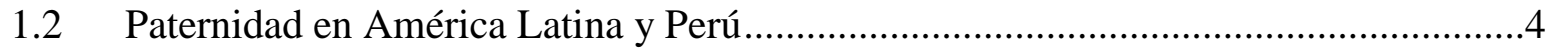

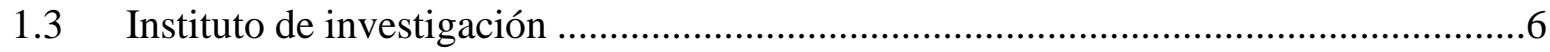

\section{CAPÍTULO II: DESCRIPCIÓN DE LAS ACTIVIDADES Y TAREAS REALIZADAS 7}

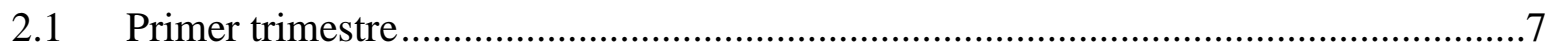

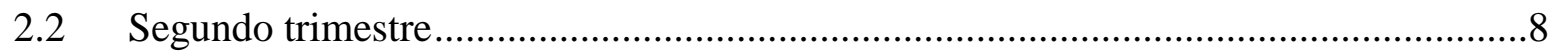

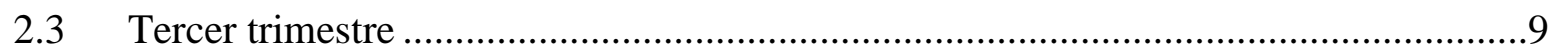

2.4 Cuarto trimestre

CAPÍTULO III: RESULTADOS DE LA INTERVENCIÓN...............................................11

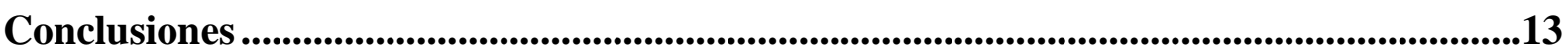

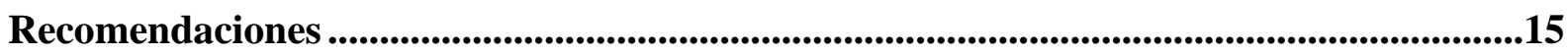

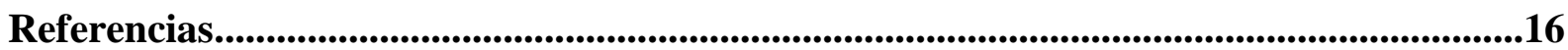

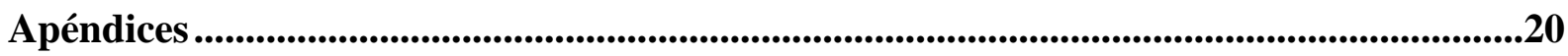




\section{ÍNDICE DE APÉNDICES}

Apéndice 1: Guía de preguntas para la primera entrevista .....................................................21 


\section{INTRODUCCIÓN}

Cuando se habla de paternidad y se realiza una revisión bibliográfica sobre esta temática se encuentra que la mayoría de estudios han explorado las transformaciones en la mujer, dejando la vivencia de los hombres como un campo aún desconocido (Miguélez, 2016). Si bien esto podría justificarse con aspectos socioculturales que ubicaban a la mujer como sinónimo de maternidad, y al hombre como proveedor económico (Puyana y Mosquera, 2005), en las últimas décadas se reporta que los padres participan más en el cuidado de los hijos, construyendo un vínculo más afectivo y ubicándolos en las llamadas "Nuevas paternidades" (Krob, Piccinini y da Rosa, 2009; Singleton y Maher, 2004).

Por tal motivo, se planteó realizar una investigación cualitativa orientada a analizar y comprender los significados y el proceso de construcción en padres primerizos de Lima Metropolitana, enfocándose en la transición hacia la nueva identidad, cambios en la relación de pareja, entre otros. Los resultados encontrados aportarían a la psicología conocimientos sobre aspectos principales que intervienen en la transición hacia la de paternidad y el impacto que tienen en el desarrollo del infante.

El presente trabajo pretende explicar de manera detallada los procedimientos realizados para la consecución de dicho objetivo. En primer lugar se hará una revisión sobre la problemática planteada, teniendo en consideración el contexto donde se encuentra y los factores que intervienen. En segundo lugar, se mencionarán las actividades realizadas a lo largo del proyecto de investigación. En tercer lugar, se realiza una revisión crítica de la intervención, teniendo en cuenta aportes, logros, estrategias utilizadas y limitaciones que se han encontrado a lo largo del estudio. Por último se da paso a las conclusiones y recomendaciones para los futuros investigadores. 


\section{CAPÍTULO I: IDENTIFICACIÓN DEL PROBLEMA}

\subsection{Paternidad tradicional VS. Nuevas paternidades}

La transición hacia la paternidad trae consigo un gran número de cambios a nivel personal y en el estilo de vida de las parejas, obligándolos a realizar grandes transformaciones que impactarán en su nuevo rol y en la relación con el bebé (Bouchard, 2012; Fillo, Simpson, Rholes y Kohn, 2015; Ohashi \& Asano, 2012). Dichos cambios afectan tanto a la madre como al padre; sin embargo, cuando se habla de paternidad, la mayoría de investigaciones han estado centradas en explorar casi de manera exclusiva las transformaciones físicas y psicológicas que experimenta la mujer y sin duda han ocupado más espacio y bibliografía que en el caso de los hombres (Miguélez, 2016). Esto se podría deber a que históricamente el papel de cuidado y crianza de los hijos se les ha atribuido a las mujeres, mientras que los hombres se encargaban de proveer económicamente a la familia (Puyana y Mosquera, 2005).

La construcción de la paternidad y maternidad está inscrita dentro de un contexto sociocultural, por tanto, este determina de qué manera deben asumir hombres y mujeres este acontecimiento, estableciendo qué sentir e incluso cómo demostrarlo públicamente, generando representaciones sobre lo que significa ser padre y/o madre (Aguayo, Barker y Kimelman, 2016; López y Ferrari, 2010;). En este sentido, al estar atravesado por lo sociocultural, genera que se reproduzcan modelos "correctos" y "esperables" de comportamientos masculinos y femeninos considerados adecuados para la época y sociedad en la que se encuentran (Aguayo et al., 2016). Por tal motivo, dichas concepciones serán cambiantes según las modificaciones sociales que vayan ocurriendo (Carvalho de Souza y Pereira da Cruz, 2009; Füller, 2000; Lupton y Barclay, 1997; Salguero, 2006; Singleton y Maher, 2004; Vieira y Nascimento, 2013). 
Desde los años 70, las influencias socioculturales han ido modificando esta visión sobre la paternidad. Tradicionalmente los hombres pasaban largas jornadas laborales para lograr cumplir con el rol de proveedor económico que se esperaba de ellos, acentuando entonces la división de tareas en base al género al que se pertenece. En este sentido, el hombre trabajaba para mantener a la familia y la mujer se encargaba del cuidado de los hijos y de la casa (López y Ferrari, 2010). Con la incorporación de la mujer al mundo laboral y su gradual salida del hogar, el hombre pasa a participar más en el área doméstica. Es así que se generan cambios en la las concepciones sobre la masculinidad y femineidad, y por consecuente, hay mayor diálogo sobre las tareas del hogar, la crianza y el impacto de los padres en la crianza de los hijos (Carvalho de Souza y Pereira da Cruz, 2009; Füller, 2000; Lupton y Barclay, 1997; Vieira y Nascimento, 2013).

A partir de los cambios señalados con anterioridad surgen los llamados "nuevos padres" (Singleton y Maher, 2004), quienes se caracterizan por tener mayor expresión de afecto e involucramiento en la crianza de los hijos. A diferencia de la paternidad tradicional matizada por el distanciamiento emocional padre-hijo, las nuevas paternidades muestran una relación definida por la sensibilidad y cercanía (Ferrari, 2011; Füller, 2000; Janto, 2015; Marcos, 2011; Vieira y Nascimento, 2013). A nivel de sociedad, se espera que el hombre sea capaz de contener emocionalmente a su pareja e hijo, brindarles amor, cuidado y seguridad, más allá de cumplir con el rol tradicional (Nieri, 2017).

Sin embrago, a pesar de la flexibilidad en los discursos sobre paternidad y las aspiraciones a vincularse más con sus hijos, aún persiste la visión en la que deben cumplir con el papel de proveedor (Izquierdo y Zicavo, 2015; Miller, 2010; Oesterheld y Pavicevic, 2016), evidenciando cómo aún predominan elementos más tradicionales en combinación con aspectos novedosos. 
Como se mencionó con anterioridad, aún son escasos los conocimientos que se tienen sobre el proceso de construcción de paternidad en hombres, y por tal motivo, es inquietante no solo conocer a mayor detalle cuáles son los beneficios del involucramiento afectivo del padre en la vida de sus hijos, sino también indagar cuáles son los beneficios para él mismo, la familia y la sociedad. Algunas investigaciones reportan que la participación de los padres tiene un impacto positivo en el desarrollo cognitivo, salud mental y bienestar socioemocional actual y posterior en el infante (Levtov, Van der Gaag, Greene, Michael y Baker, 2015; Sarkadi, Kristiansson, Oberklaid y Bremberg, 2008; Shaffer y Kipp, 2013;). En el caso de los padres, estos reportan que se sienten más realizados y suelen cuidar más de su salud, presentando menores niveles de estrés, enfermedades y vida más longeva y satisfactoria. De la misma manera, a nivel de sociedad, una paternidad responsable contribuye a lograr la equidad de género (Barker y Verani, 2008).

Dicho esto, constituye un aporte para la psicología explorar y comprender los aspectos principales que intervienen en la paternidad para profundizar más en el impacto que tienen en el infante y para comprender la transformación hacia la nueva identidad.

\subsection{Paternidad en América Latina y Perú}

En América Latina el número de padres interesados en participar en la crianza de sus hijos va en aumento. Sin embrago, teniendo en consideración que la mayoría de países aún tiene sociedades con actitudes machistas, se refuerza y prolonga que la aproximación de los hombres al cuidado de los hijos sea poca debido a los roles dominantes de la paternidad y la división del trabajo (Aguayo et. al, 2016). Olavarría (2001), menciona que para lograr una equilibro entre el hombre, la vida familiar y laboral, el estado y la 
sociedad, es necesario que se generen más programas y políticas públicas orientadas a lograr la equidad de género.

Adentrándonos en la realidad nacional, en 2013 el Instituto Nacional de Estadística e Informática (INEI, 2014) reportó que el 26\% de las madres se encargan solas del cuidado de los hijos, aumentando a $28 \%$ en zonas urbanas. Esto da cuenta de cómo en nuestra sociedad resulta difícil desarrollar la paternidad. Füller (2000), es quien ha realizado en el Perú un estudio en hombres que ha abarcado los departamentos de Lima, Cusco e Iquitos, con la finalidad de conocer los ideales de paternidad de dichos participantes. Como principales hallazgos encontró que esta vivencia es descrita como una experiencia transformadora que implica renunciar a la individualidad y comprometerse de manera económica y afectiva con la pareja y futuros hijos. Este último punto tiene especial importancia ya que en Perú son muchas madres quienes asumen la jefatura familiar por consecuencia del abandono o ausencia paterna.

En Lima, Marcos (2011) realizó una investigación con padres adolescentes de sectores socioeconómicos bajos, encontrando que tienen una relación cercana con sus hijos, y a su vez, continúan presentando concepciones tradicionales de la paternidad en la que deben cumplir con el papel de proveer económicamente. Bajo esta línea se pueden encontrar dificultades para cumplir con ambos roles, ya que por un lado buscan vincularse afectivamente, pero también se ven limitados por tener que cumplir con extensas jornadas laborales que podrían dificultar el compromiso y participación de estos padres en la vida de sus hijos.

Como se puede observar, son pocos los alcances de investigación realizados sobre paternidad a nivel mundial, y más aún en nuestro contexto, por lo que se plantea continuar investigando para explorar más sobre este fenómeno. Al empezar a entender 
esta realidad, se podrán implementar políticas y nuevos modelos de comportamiento que promuevan la participación de los padres.

A partir de lo expuesto, se planteó un estudio cualitativo orientado a conocer cómo es la vivencia de paternidad en padres primerizos de Lima Metropolitana.

\subsection{Instituto de investigación}

Tras revisar la problemática planteada, se presentó dicha propuesta a un instituto de investigación, unidad encargada de promover, gestionar y coordinar la producción científica de los docentes universitarios. Con miras a impulsar la investigación en la comunidad académica, esta entidad informa a la comunidad universitaria sobre los proyectos presentados por los profesores, buscando que los hallazgos logren contribuir, a través de la creación de nuevos conocimientos, al desarrollo del país.

Una vez culminados los proyectos, evalúa los trabajos y sugiere su publicación en fondos editoriales o en revistas especializadas. De esta manera, las investigaciones fortalecerán los vínculos con otras universidades y centros de investigación nacionales e internacionales. 


\section{CAPÍTULO II: DESCRIPCIÓN DE LAS ACTIVIDADES Y TAREAS REALIZADAS}

El plan de trabajo establecido se proyectó para 12 meses, en relación a los objetivos y entregas trimestrales al Instituto. Cada trimestre se reportó al instituto un avance. A continuación, se describe las actividades realizadas en cada trimestre

\subsection{Primer trimestre}

Durante el primer trimestre, las actividades principales estuvieron orientadas a la búsqueda bibliográfica para la elaboración del marco teórico y del método.

Se revisaron distintas fuentes utilizando como criterio que sean publicaciones de actualidad y en revistas con rigor científico e indexadas. La investigación fue planteada bajo el método cualitativo, con carácter fenomenológico, orientado a conocer, comprender y describir la vivencia de este fenómeno desde la perspectiva de padres primerizos de Lima Metropolitana (Bogdan y Biklen, 2007; Hernández, Fernández y Baptista, 2014). A su vez, se realizó un estudio de casos múltiple para examinar de manera detallada cómo se presenta este fenómeno a partir del seguimiento a ocho padres en relación a la paternidad. De esta manera, se podrán encontrar aspectos particulares y diversos que evidencien la complejidad del fenómeno y que permitan dar respuesta a la pregunta de investigación (Yin, 2017).

Se propuso, durante la elaboración del método, los criterios de inclusión para la muestra, dichos padres debían ser primerizos, pertenecer al nivel socioeconómico A o B y que provengan de situaciones familiares que oscilen entre 1) vivir con su pareja y bebé y trabajar dentro de casa bajo la modalidad de Homeoffice o Independiente, o 2) vivir con su pareja y bebé, laborando fuera de casa. A su vez, se estableció la realización de dos 
entrevistas a lo largo del estudio. La primera debía ser realizada dentro de los primeros 6 meses del bebé, y la segunda cuando ya estuviera próximo a cumplir un año. De esta manera se podrían explorar los cambios intrapsíquicos ocurridos en cada participante desde el momento del embarazo hasta cuando comienzan a interactuar más con el bebé.

\subsection{Segundo trimestre}

A partir de la revisión bibliográfica mencionada con anterioridad, se logró identificar qué variables influenciaban el proceso de paternidad, hecho que facilitó la elaboración de la Guía de preguntas (Apéndice 1) para las entrevistas y la Ficha de datos, que serían utilizados como método de recolección de datos; y a su vez, proporcionó información necesaria para el posterior análisis de las entrevistas. Una vez construidos ambos materiales, se procedió a realizar una entrevista piloto con la finalidad de revisar el correcto funcionamiento de las preguntas y explorar la duración aproximada de la entrevista.

Luego se inició el contacto con los participantes bajo el método de bola de nieve. Teniendo en cuenta las características del estudio, se realizó un muestreo de tipo probabilístico e intencional. La muestra consistió en 8 padres primerizos de Lima Metropolitana, encontrándose entre los 26 y 44 años de edad, pertenecientes al nivel socioeconómico A o B. La mayoría de ellos se encuentra trabajando fuera del hogar (7/8) y casi todos deseaban ser padres (7/8). Todos los participantes viven actualmente con su pareja y/o esposa e hijo/a.

Llegado el momento de la primera entrevista, se procedió a explicar de manera oral y escrita el consentimiento informado, asegurando en todo momento la seguridad y anonimato de los padres participantes. Luego de cada una, se procedió a transcribirlas y a 
eliminar inmediatamente las grabaciones. Tanto los nombres de los padres como el de los bebés y su pareja y/o esposa fueron modificados.

Esta entrevista estuvo centrada en conocer el impacto de la noticia de la llegada del bebé, actitudes respecto al rol paterno, la relación con su propio padre, cambios generados en la dinámica de pareja, entre otros. Se elaboró posteriormente un análisis preliminar de las mismas, creando categorías de análisis que abarquen de manera transversal las temáticas más relevantes.

\subsection{Tercer trimestre}

Luego de tres meses se realizaron las segundas entrevistas correspondientes. Para este caso, existían preguntas transversales para todos los padres y otras diferenciadas en base a la información obtenida en la primera entrevista. La temática se orientó en explorar qué cambios habían percibido en sus bebés y en ellos mismos desde la última entrevista, teniendo en consideración que desde el aspecto evolutivo del niño comienzan a ser más responsivos con el medio, por lo que empiezan a interactuar más con el padre, y de qué manera esto puede haber impactado en la relación con ellos. A su vez se quiso explorar si se percibieron cambios en la relación de pareja y en la distribución de tareas y/o actividades con el bebé.

Al igual que en la primera entrevista, se transcribió y eliminó la grabación de cada una. Luego se procedió a construir las categorías de análisis para estas entrevistas y se realizó un segundo análisis preliminar de las mismas.

\subsection{Cuarto trimestre}


En el último trimestre se integraron los resultados de ambas entrevistas y se elaboró la discusión de los mismos. También se procedió a buscar mayor bibliografía que permita un análisis más profundo de las categorías construidas.

Teniendo en cuenta la extensión de la discusión, se procedió a dividir los hallazgos en dos grupos. El primero de ellos, contenía información relacionada al proceso de paternidad y su construcción, indagando en los cambios ocurridos en la identidad del padre, articulación de la pareja, relación con el bebé, aspecto laboral y revisión de la masculinidad en el periodo pre y perinatal. Para el segundo grupo, se quiso incidir más en el nacimiento del bebé como acontecimiento que marca el inicio de la paternidad y en explorar los cambios intrapsíquicos que ocurren en el padre cuando el bebé crece y comienza a vincularse más con él. De esta manera, se buscó realizar dos publicaciones en vez de una, logrando hacer un análisis más profundo de las variables.

Aún se encuentra en proceso la redacción de las conclusiones. A finales del mes de marzo se entregará al instituto ambas investigaciones finalizadas y listas para publicarlas en las revistas elegidas por los investigadores. 


\section{CAPÍTULO III: RESULTADOS DE LA INTERVENCIÓN}

El estudio descrito buscó analizar y comprender cómo es la transición hacia la paternidad en padres primerizos de Lima Metropolitana y cómo se aproximan a esta nueva tarea, teniendo en cuenta los cambios ocurridos desde el anuncio del embarazo hasta antes del primer año de vida.

Utilizando el estudio de casos múltiple, se logró una comprensión más rica y profunda de la vivencia de paternidad. Al tener en cuenta que se buscaban situaciones familiares distintas en los padres (que oscilen entre trabajar dentro o fuera del hogar), se pudo recoger la particularidad de estas experiencias con sus elementos propios y comunes. Esta selección de casos diferenciados, mencionan Flores, Gómez y Jiménez (1999), aporta criterios complementarios al estudio, permitiendo observar la variedad y particularidad de este fenómeno en cada padre, sin olvidar el equilibro existente por las características comunes que comparten estos participantes. En cuanto a los métodos de recolección de datos, la Ficha de datos permitió recoger información básica, documento que facilitó que el investigador pudiera acceder a dichos datos de manera rápida, sencilla y ordenada. Sobre las entrevistas a profundidad, la escasa estructuración permitió que los participantes sean quienes guíen la información brindada y manifiesten de manera particular y diferenciada su propia experiencia. A su vez, la realización de dos entrevistas en distintos momentos aportó un análisis más detallado que permitió, por un lado, conocer el tránsito y el proceso hacia la construcción de la nueva identidad, así como también, explorar los cambios percibidos en ellos, el bebé y en la interacción entre ambos cuando el bebé se encuentra próximo a cumplir un año.

La combinación de los métodos mencionados anteriormente permitió recopilar información profunda sobre la vivencia de la paternidad en estos padres primerizos, e incluso permitió que 
se construyera material suficiente para la próxima publicación de dos investigaciones que generarán más temas de investigación.

La obtención de dichos resultados se vio beneficiado por las entregas trimestrales que el Instituto de investigación pedía a los investigadores, ya que propiciaba la organización y optimización del tiempo para cumplir dichas metas. A lo largo del estudio, la totalidad de las entregas fueron dentro del lapso determinado, e incluso el análisis preliminar de las primeras entrevistas fue antes de las fechas establecidas.

A partir de la investigación se logró generar conocimiento en un campo poco indagado por los aspectos socioculturales mencionados a lo largo del trabajo. Se encontró como principales descubrimientos que la paternidad, al igual que ocurre en la madre, es una experiencia interna impactante que genera una serie de cambios en el próximo padre y que influirá en su nueva identidad y en el futuro vínculo con su hijo. Asimismo, se encontró que el factor de la edad es un aspecto relevante a la hora de convertirse en padre. Se halló que los mayores son quienes tienen mayor facilidad para adaptarse a los nuevos roles, a diferencia de los más jóvenes quienes presentaban actitudes correspondientes a una paternidad más tradicional centrada en el rol de proveer.

Las principales limitaciones estuvieron relacionadas a la muestra. Por un lado, la especificidad de los participantes dificultó poder encontrar rápido a los padres, ya que debían cumplir los criterios previamente establecidos en el método. También, hubo dificultades en el seguimiento a los padres a la hora de realizar las segundas entrevistas, ya que por motivos laborales o familiares resultada difícil concretar un encuentro. Este último punto resultaba de gran importancia ya que podía poner en riesgo la continuidad de la investigación y o afectarla de manera negativa. A pesar de las dificultades, la investigación pudo proceder sin mayores complicaciones. 


\section{CONCLUSIONES}

- La paternidad es una experiencia movilizante que genera una serie de cambios en la identidad del futuro padre. Según la manera en que sucedan dichos reacomodos intrapsíquicos, tendrá diferente impacto en el vínculo con su futuro bebé y en su desenvolvimiento en el papel de padre.

- Teniendo en cuenta el dinamismo de la realidad humana y las limitaciones del modelo tradicional para investigar esta realidad, se incide en la importancia de utilizar la metodología cualitativa, ya que a partir de la aproximación interpretativa se podrán conocer las representaciones que tienen los padres primerizos sobre la paternidad y todos los cambios que este conlleva, teniendo en consideración la influencia del contexto sociocultural que impacta en la manera de aproximarse a este rol.

- La selección adecuada de la metodología para explorar este fenómeno, ha permitido que se obtengan resultados profundos, incluso al punto de poder realizar dos publicaciones en vez de una. Es por ello que se incide en la importancia de formular y utilizar de manera adecuada aquellas herramientas que permitan generar hallazgos novedosos que puedan aportar a la psicología.

- En este caso, los resultados de la investigación constituirían un aporte novedoso con respecto a la vivencia de la paternidad y la nueva construcción de identidad, ya que usualmente se ha explorado este aspecto en madres, debido a que se asume que son ellas las encargadas de ejercer el rol de cuidado en los hijos.

- A partir de los hallazgos en el estudio, se podrán generar nuevas líneas de investigación, tales como explorar el proceso de transición en: padres que se encuentren en proceso de adopción, hayan pasado por un tratamiento de fertilidad, pertenecientes a todos niveles socioeconómicos o que actualmente no vivan con sus 
hijos, de manera que se profundice más en este fenómeno y se pueda generar mayor conocimiento. 


\section{RECOMENDACIONES}

A continuación se detallaran las recomendaciones:

$\checkmark$ Prestar atención a las fuentes que se revisan para el estudio, ya que estas serán la base para construir la introducción, métodos y posterior análisis. Tomar en cuenta que sean recientes, se encuentren en revistas indexadas, etc.

$\checkmark$ Realizar un cronograma con metas semanales y/o mensuales, de manera que se aproveche más el tiempo en base a los objetivos propuestos

$\checkmark$ Realizar cuadros-resumen para las investigaciones que se van revisando, pueden incluir divisiones por autor/es, año de publicación, tipo de investigación y una breve descripción de la investigación. De esta manera se podrá revisar información relevante de cada una de ellas optimizando tiempo.

$\checkmark$ En el caso de une investigación cualitativa, es indispensable realizar un piloto del método de recolección de datos que se vaya a utilizar, ya sea entrevista, focus grupo, etc., y que este mismo sea revisado por especialistas. Así, se podrá garantizar que la formulación de preguntas sea la adecuada, probar las destrezas del investigador y la extensión de la entrevista.

$\checkmark$ A la hora de publicar, tener en cuenta las consideraciones que tiene cada revista para su publicación. Se sugiere que sea revisado con antelación para tener tiempo suficiente para realizar las modificaciones respectivas.

$\checkmark$ Buscar revistas que sean adecuadas al tema que se quiere presentar. Por ejemplo, si la temática es sobre paternidad, buscar revistas o ediciones que traten de manera específica estos temas. 


\section{REFERENCIAS}

Aguayo, F., Barker, G., y Kimelman, E. (2016). Paternidad y cuidado en América Latina: Ausencias, presencias y transformaciones. Masculinities \& Social Change, 5(2), 98106. http://doi.org/10.17583/MCS.2016.2140

Barker, G. y Verani, F. (2008). La participación del hombre como padre en la región de Latinoamérica y el Caribe: Una revisión de literatura crítica con consideraciones para políticas. Brasil: Instituto Promundo.

Bogdan, R y Biklen, S. (2007) Qualitative Research for Education: An Introduction to Theories and Methods ( $5^{\mathrm{a}}$ ed). Estados Unidos: Pearson.

Bouchard, G. (2012). Intergenerational transmission and transition to fatherhood: A mediated-moderation model of paternal engagement. Journal of Family Psychology, 26(5), 747-755.

Carvalho de Souza, C., y Pereira da Cruz Benetti, S. (2009). Paternidade contemporânea: levantamento da produção acadêmica no período de 2000 a 2007. Paidéia, 19(42), 97 106. Recuperado de https://www.redalyc.org/pdf/3054/305423764012.pdf

Ferrari, J. (2011). Padre amado o deseado. La nueva relación entre padres e hijos. México: Trillas.

Fillo, J., Simpson, J. A., Rholes, W. S., y Kohn, J. L. (2015). Dads doing diapers: Individual and relational outcomes associated with the division of child care across the transition to parenthood. Journal of Personality and Social Psychology, 108, 298-316. http://dx.doi.org/10.1037/a0038572

Flores, J. G., Gómez, G. R., y Jiménez, E. G. (1999). Metodología de la investigación cualitativa. Málaga, España: Aljibe. 
Füller, N. (2000). Significados y prácticas de paternidad entre varones urbanos del Perú. Paternidades en América Latina, 35-90.

Hernández, R., Fernández, C. y Baptista, P. (2014). Metodología de la investigación (6 ${ }^{\mathrm{a}}$ ed.). México D.F.: McGraw-Hill.

Instituto Nacional de Estadística e Informática (2014). Estado de la Población Peruana 2014. Recuperado de https://www.inei.gob.pe/media/MenuRecursivo/publicaciones_digitales/Est/Lib1251/ Libro.pdf

Izquierdo, L., y Zicavo, N. (2015). Nuevos padres: construcción del rol paternal en hombres que participan activamente en la crianza de los hijos. Revista de Investigación en Psicología, 18(2), 33-55. http://dx.doi.org/10.15381/rinvp.v18i2.12082

Janto, C. (2015). La paternidad: una creación compartida. Descubrimiento y construcción de la paternidad en ocho padres primerizos de Lima. Tesis de Licenciatura, Pontificia Universidad Católica del Perú, Lima.

Krob, A. D., Piccinini, C. A., y Silva, M. R. (2009). A transição para a paternidade: da gestação ao segundo mês de vida do bebê. Psicologia USP, 20(2), 269-291. https://doi.org/10.1590/S0103-65642009000200008

Levtov, R., Van der Gaag, N., Greene, M., Michael, K., y Barker, G. (2015). State of the World's Fathers: A MenCare Advocacy Publication. Washington, DC: Promundo.

López, P., y Ferrari, F. (2010). Apuntes para el trabajo en sexualidad desde los enfoques de género, derechos y diversidad. Uruguay: Gurises Unidos.

Lupton, D. y Barclay, L. (1997). Constructing Fatherhood, Discourses and experiences. London: Sage publications. 
Marcos, C. (2011). Representaciones mentales de la paternidad en padres varones adolescentes. Tesis de Licenciatura, Pontificia Universidad Católica del Perú, Lima.

Miguélez, A. A. (2016). Negociaciones de pareja: los trabajos domésticos, la crianza y la construcción de la maternidad y la paternidad. Papeles del CEIC. International Journal on Collective Identity Research, (1), 1-27. http://dx.doi.org/10.1387/pceic.15209

Miller, T. (2010). Making sense of fatherhood: Gender, caring and work. Cambridge University Press.

Nieri, L. (2017). Transición y construcción de la paternidad. Interdisciplinaria: Revista de psicología y ciencias afines, (2), 425-440.

Oesterheld, F. H., y Pavicevic, Y. (2016). Anticipando la Paternidad: Ella es la que está embarazada. Masculinidades $\quad y \quad$ cambio $\quad$ social, 5(2), 107-133. http://doi.org/10.17583/MCS.2016.2038

Ohashi, Y., y Asano, M. (2012). Transition to early parenthood, and family functioning relationships in Japan: a longitudinal study. Nursing and Health Sciences, 14, 140147. http://dx.doi.org/10.1111/j.1442-2018.2011.00669.x

Olavarría, J. (2001). ¿Hombres a la deriva? Poder, trabajo y sexo. Santiago: FLASCO

Puyana Villamizar, Y., y Mosquera Rosero, C. (2005). Traer" hijos o hijas al mundo": significados culturales de la paternidad y la maternidad. Revista latinoamericana de Ciencias sociales, Niñez y Juventud, 3(2), 111-140. Recuperado de http://www.scielo.org.co/pdf/rlcs/v3n2/v3n2a05.pdf 
Salguero, M. (2006). Significado y vivencia de la paternidad en algunos varones de los sectores socioeconómicos medios en la Ciudad de México. Ser padres, esposos e hijos: prácticas y valoraciones de varones mexicanos, 57-94.

Sarkadi, A., Kristiansson, R., Oberklaid, F., y Bremberg, S. (2008). Fathers' involvement and children's developmental outcomes: a systematic review of longitudinal studies. Acta paediatrica, 97(2), 153-158. https://doi.org/10.1111/j.1651-2227.2007.00572.x

Shaffer, D. R., y Kipp, K. (2013). Developmental psychology: Childhood and adolescence (9a ed). Cengage Learning.

Singleton, A., y Maher, J. (2004). The "new man" is in the house: Young men, social change, and housework. The Journal of Men's Studies, 12(3), 227-240. https://doi.org/10.3149/jms.1203.227

Vieira, G. T., y Nascimento, A. R. A. (2013). Aspectos psicossociais da construção da identidade paterna. Revista Psicologia-Teoria e Prática,16(1), 57-68. . http://dx.doi.org/10.15348/1980-6906/psicologia.v16n1p57-68

Yin, R. K. (2017). Case study research and applications: Design and methods (6 $6^{\mathrm{a}}$ ed). Estados Unidos: Sage publications. 


\section{APÉNDICES}




\section{APÉNDICE 1: GUÍA DE PREGUNTAS PARA LA PRIMERA ENTREVISTA}

\section{$\underline{\text { Guía de preguntas }}$}

Buenos días/tardes (nombre), desde ya le agradecemos su participación. Como se explicó en el consentimiento, el proceso consiste en dos entrevistas y en cada oportunidad el tiempo de duración será aproximadamente 50-60 minutos. Lo conversado en estas sesiones será grabado con la finalidad de poder transcribir las ideas que hayas expresado. Tus respuestas serán codificadas usando un número de identificación, por lo tanto, serán anónimas y toda información que se recoja será confidencial.

Ante cualquier duda, puede hacer preguntas en cualquier momento de la participación. De la misma manera, si alguna de las preguntas durante la entrevista le parecen incómodas tiene derecho de hacérselo saber al investigador o de no responderlas.

Cuando eras joven, ¿Te habías imaginado siendo papá antes?, ¿Cómo era el rol, en qué situación y hace cuánto tiempo?

Con respecto a la mamá de tu hijo/a, ¿Hubo una idea previa de ser papás?, ¿Cómo así surge la idea?

¿Cómo fue la noticia?, ¿Qué pensaste cuando te enteraste que iba a ser hombre/mujer?

¿Cómo viviste esta próxima paternidad?, ¿Cómo viviste el saber que ibas a ser papá durante el embarazo?

¿Cómo ha cambiado tu vida?

¿Cómo era la dinámica con tu pareja?, ¿Ha evolucionado o presentado cambios?

Con respecto a los primeros meses del bebé ¿Cómo fue la vivencia?, ¿Qué actividades compartían? ¿Cuál era tu rol?

¿Cómo visualizas la relación con tu hijo/a cuando crezca?

¿Cuáles son tus expectativas en relación a tu hijo/hija?

¿Cómo construyen con la mamá del niño/a la paternidad?

¿Cómo era la dinámica con tu papá?, ¿Y con tu mamá?, ¿Qué hacías con cada uno?, ¿Qué recuerdos tienes de tu primera infancia? Si no hubo papa, ¿Quién cumplió ese rol y cómo fue la dinámica?

¿Qué es lo que más valoras de ser papá?, ¿Qué es lo más difícil? 